\title{
EL PROCESO DE INSTITUCIONALIZACIÓN DE LA EXPERIENCIA COOPERATIVA MONDRAGÓN
}

\author{
The institutionalization process \\ of the Mondragon cooperative experience \\ Rafael Altuna*1, Eguzki Urteaga ${ }^{2}$ \\ ${ }^{1}$ Mondragon Unibertsitatea, Facultad de Empresariales \\ 2 Universidad del País Vasco / Euskal Herriko Unibertsitatea
}

\section{RESUMEN}

El presente artículo explica los rasgos institucionales que han caracterizado a la Experiencia Cooperativa Mondragón (ECM) desde su creación y los elementos institucionales más relevantes que la constituyen. Para ello, analiza las bases sobre las que se apoya la ECM en su fundación y que le van a servir de elemento de legitimación durante sus primeros años de existencia. Estas bases, que son denominadas por la teoría neoinstitucional (normativas, reguladoras y cognitivas), le proporcionan estabilidad y significado a lo largo del tiempo. De este modo, ECM se constituye en patrones culturales que proporcionan medios y valores compartidos a sus estructuras y labores particulares, integrándolas en grandes proyectos.

Palabras clave: institucionalización - experiencia cooperativa - cooperativas de Mondragón.

\section{ABSTRACT}

The present article analyzes the institutional characteristics of the Mondragon's Cooperative Experience (MCE) since its origin and the most relevant institutional elements that constitute it. For that, it analyzes the bases on which the ECM is sustained in its origin and which are going to use it as element of legitimization during the first decades of existence. These bases, which are named by the theory neoinstitucional (normative, regulatory and cognitive), provide stability and meaning to it throughout the time. MCE is constituted in cultural procedures that provide resources and values to its entities and particular activities integrating them in big projects.

Keywords: institutionalization - cooperative experience - Mondragón's cooperatives.

* Correspondencia a: Rafael Altuna. Mondragon Unibertsitatea. Enpresagintza. Ibarra Zelaia 2. Oñati. 20560 Gipuzkoa - raltuna@ mondragon.edu - https://orcid.org/0000-0001-8112-9815

Cómo citar: Altuna, Rafael; Urteaga, Eguzki. (2018). «El proceso de institucionalización de la experiencia cooperativa Mondragón»; Lan Harremanak, 40, 138-162. (https://doi.org/10.1387/lan-harremanak.20277).

Recibido: 31 octubre, 2018; aceptado: 3 diciembre, 2018.

ISSN 1575-7048 - eISSN 2444-5819 / (C) 2018 UPV/EHU

(c) Esta obra está bajo una licencia

Creative Commons Atribución 4.0 Internacional 


\section{SUMARIO}

1. Introducción.-2. Los elementos simbólico-culturales para una base cognitiva común. 2.1. Situación de pobreza y necesidad extrema. 2.2. Una temprana industrialización. 2.3. Conciencia nacional vasca. 2.4. Casa, grupo doméstico y ámbitos de sociabilidad comunitaria. 2.5. La Iglesia como institución social central de la vida social._-3. La creación de un discurso moral generador de una base normativa propia. 3.1. La persona y la doctrina social de la Iglesia. 3.2. La cooperación. 3.3. Desarrollo comunitario. 3.4. Acción desde el pragmatismo.-4. Las bases reguladoras e innovadoras al servicio del proyecto socio-empresarial. 4.1. Reglas relativas al tratamiento del capital. 4.2. Reglas relativas al gobierno.-5. Conclusión.-6. Bibliografía.

\section{Introducción}

Comprender la realidad actual de la Experiencia Cooperativa Mondragón (ECM) requiere entender su origen y evolución, dado que, tal y como afirma Perrow (1991: 191), «el presente está enraizado en el pasado». Es éste un proceso continuado de institucionalización, esto es, «el proceso a través del cual, los componentes de una estructura formal se vuelven ampliamente aceptados como apropiados y necesarios, y sirven para legitimar a las organizaciones» (Tolbert y Zucker, 1983: 25).

La ECM es una realidad institucionalizada, esto es una realidad en continuo proceso de construcción social, resultado de las interacciones entre múltiples actores, grupos y organizaciones, tendentes al consenso, pero no exentas de divergencias, presiones, y conflictos, en contextos sociales, económicos y políticos que generan una creciente incertidumbre. El resultado de todo ello es un conjunto identificable de sistemas de significado y patrones de comportamiento, de reglas constitutivas y normativas, y de procesos regulativos identificables externamente.

La ECM en los diferentes momentos históricos de su evolución, ha tenido que dar respuesta a las demandas que le han realizado tanto el entorno técnico como el institucional. Habitualmente, los análisis organizativos inciden en los aspectos técnicos pero, con frecuencia, desestiman los aspectos institucionales. Sin embargo, ambos son complementarios en la medida en que ambos contribuyen al logro de la supervivencia de la organización. Las demandas tanto técnicas como institucionales evolucionan con el tiempo en la medida en que el 
entorno técnico e institucional también se modifica. Es por ello que las organizaciones han de permanecer atentas a ambos entornos para interpretarlos de manera adecuada.

En la medida en que la Experiencia Cooperativa Mondragón logra dar respuesta a las demandas institucionales, su legitimidad se verá incrementada y, de esta manera, también logrará mejores recursos de todo orden para su desenvolvimiento. Por esta razón, la búsqueda de legitimidad se vuelve un elemento de primer orden para el logro de la supervivencia organizativa. La legitimidad es un rasgo conferido por los actores sociales, por lo que la acción legítima será aquélla cuyos valores y expectativas de acción reflejan y coinciden con las de los actores (Galaskiewcs, 1985). Dado que éstos son diversos y puesto que sus valores y expectativas difieren en el tiempo, las organizaciones se ven obligadas a adecuar su acción en el tiempo.

El presente texto da cuenta de las características institucionales de la ECM desde su origen y los componentes institucionales más relevantes que lo conforman. Para iniciarlo, analizaremos las bases sobre las que se sustenta la ECM en su creación y que la legitimarán durante sus primeras décadas de vida. Estas bases son las «estructuras cognitivas, normativas y reguladoras que le proporcionan estabilidad y significado" a lo largo del tiempo (Scott, 1995: 33). De este modo, las instituciones, siguiendo a Scott, se constituyen en normas culturales que proporcionan recursos y valores compartidos a las entidades y a las ocupaciones específicas integrándolas en grandes proyectos. Las instituciones, por tanto, son sistemas de significado y modelos de comportamiento que contienen elementos simbólicos, reforzados por procesos reguladores.

\section{Los elementos simbólico-culturales para una base cognitiva común}

La teoría neoinstitucional entiende el pilar cognitivo como las reglas que constituyen la naturaleza de la realidad y las fórmulas a través de las cuales se elabora el significado. Los símbolos (palabras, signos y gestos) determinan el significado que los individuos atribuyen a los objetos y las acciones. Se hace necesario que las bases normativas de una determinada realidad social, en este caso la ECM, tenga coherencia con los fundamentos cognitivos de los individuos que la integran. De lo contrario, los principios normativos carecerían de significación para los individuos, no pudiendo ser subjetivadas. El pilar normativo, por tanto, tiene base cultural, siendo ésta, fuente de legitimidad.

Para entender las bases cognitivas de la ECM, hemos de referirnos a aspectos de carácter geográfico, político, histórico, económico y cultural de la comunidad en la que se inserta dicho fenómeno institucional y que le nutre de elementos de significado que van a ser asumidos y reelaborados en un nuevo contexto institu- 
cional en ciernes. Destacaré los aspectos que han tenido mayor incidencia en la ECM.

\subsection{Situación de pobreza y necesidad extrema}

Con el inicio de la Guerra Civil (1936-1939), se situó en Mondragón un frente de lucha. Una vez finalizada, la miseria económica, la división social y el desánimo moral fueron una constante entre la población. Durante los ańos 40 y comienzos de los años 50, el hambre y la no satisfacción de las necesidades básicas, son la realidad cotidiana para la gran mayoría de la población (González y Garmendia:, 1988).

Esta situación, si bien no tan extrema, no era en absoluto nueva para la población, ya que la necesidad y la situación de pobreza se fueron alargando en buena parte del siglo xIx y comienzos del siglo xx. Las Guerras Carlistas incidieron muy negativamente en el sector primario y la emigración a América fue una alternativa muy común para los jóvenes de aquella época, lo que trajo consigo un empobrecimiento aun mayor de las zonas rurales (Barruso et. all, 2005; Ugalde, 1997). Es preciso señalar también que las condiciones geográficas y climatológicas no han sido las más adecuadas para el desarrollo de la agricultura al tratarse de valles estrechos, con una climatología adversa, dando lugar a una agricultura de montańa.

\subsection{Una temprana industrialización}

La siderurgia ha tenido históricamente una presencia relevante en la zona de Mondragón desde el siglo XVI (Azpiazu, 1999, 2002). A través de estos trabajos se desprende que la economía local no estaba sustentada exclusivamente en la agricultura, orientada al autoconsumo, siempre insuficiente y de carácter autárquico, sino que era estaba complementado de una manera sustancial a través de actividades metalúrgicas. Este hecho trajo consigo el desarrollo de una tempana cultura industrial. A lo largo de los siglos XVI y XVII el municipio se enriquece de forma importante, gracias a la industria del hierro. La industria de la armería cobra gran importancia, quien suministra espadas de acero («raya») elaboradas en las «masuquerías» en las ferrerías con el mineral de hierro del monte Udalatx. El acero de Mondragón fue muy conocido y famoso a nivel internacional. Azpiazu (2002: 17) habla de una actividad cooperativa precoz: «Una formidable área industrial. Ésta abarcaría varias decenas de villas y lugares, organizados bajo un estilo netamente cooperativo, en cuyo funcionamiento cada población, cada gremio, cada oficio, encadenaba una serie de labores y servicios».

Para finales del siglo XIX se adoptaron modelos de producción industrial, manteniéndose la tradición metalúrgica en la fabricación de artículos de cerrajería y ferretería. En este periodo surgió la empresa Unión Cerrajera (1906), máximo exponente de esta rama industrial a nivel del País Vasco hasta bien en- 
trado el siglo xx (González García, 2005). El desarrollo y evolución de esta empresa marcó el desarrollo económico y social de Mondragón durante la primera mitad del siglo xx.

A partir de los años 50, Mondragón conoció la llegada de una población emigrante masiva de otras zonas del Estado, que va a modificar profundamente su realidad social, cultural y económica. Este hecho contribuyó a conformar una identidad marcadamente obrera. Esta, no obstante, ya estaba presente desde comienzos de la década, lo que se puso de manifiesto en acontecimientos tales como: las huelgas obreras de $1916^{1}$ (Parra y Aramendia, 2004); la Revolución de Octubre de 1934, dando lugar a graves enfrentamientos sociales ${ }^{2}$ (Oktubre Taldea, 2003); y los conflictos de los años 1955-56, en cuyo contexto se crea la primera cooperativa de Mondragón.

Los inicios de la presencia socialista organizada en Mondragón se remontan a 1919, año en el que se constituye el Sindicato Libre Profesional de Obreros Metalúrgicos que formará parte de la Federación Nacional de Obreros Metalúrgicos y de la Unión General de Trabajadores (U.G.T.). Pero es a partir de 1924, cuando las ideas socialistas extienden su influencia, teniendo siempre como base el Sindicato Metalúrgico. La agrupación socialista se creó en los años 19271928 y en el año 1930 se crea la Casa del Pueblo de Mondragón, siendo la tercera más antigua de Gipuzkoa. En estos primeros años de sindicalismo local, la UGT era el sindicato mayoritario en las empresas locales y también en la Unión Cerrajera. (Oktubre Taldea, 2003).

\subsection{Conciencia nacional vasca}

Mondragón ha sido históricamente una población con una marcada conciencia nacional vasca que ha influido en todos los ámbitos de la vida social a lo largo del siglo xx. Así, el Batzoki del Partido Nacionalista Vasco se fundó en el año 1908, logrando la alcaldía el año 1912. Hasta entonces, la vida municipal estaba dominada por las ideologías liberal y sobre todo carlista, de marcado carácter tra-

\footnotetext{
${ }^{1}$ Este conflicto se desarrolló a lo largo de tres meses, siendo derrotadas las demandas de los sindicatos obreros. Cuando los trabajadores volvieron a sus puestos, la dirección de la Unión Cerrajeta se negó a readmitir a los 33 supuestos cabecillas, lo cual significaba que los despedidos tampoco podrían encontrar trabajo en ninguna de las cuarenta fábricas y talleres de la zona, porque las empresas mantenían una lista negra común para excluir a los « alborotadores » (Parra y Aramendia, 2004).

2 En octubre de 1934, se produjo una revuelta enmarcada en una convocatoria revolucionaria que tuvo lugar entre los días 5 y 19 de dicho mes, contra el gobierno radical-cedista de la II República, impulsada por el sindicato socialista UGT, el PSOE y las Juventudes Socialistas. Esta convocatoria, a nivel del País Vasco tuvo una importante repercusión, sobre todo en la zona minera de Bizkaia, que se saldó con la muerte de 40 trabajadores. En Mondragón y su comarca, este conato revolucionario se saldó con cuatro muertos (entre ellos dos directivos de la empresa Unión Cerrajera), 230 detenidos y catorce personas que escaparon a Francia (Oktubre Taldea, 1987).
} 
dicionalista. Algunos de los valores más relevantes de estos últimos, quedarán integrados en el incipiente nacionalismo jeltzale a partir de comienzos del siglo xx.

A partir de los años 60, Mondragón se va a significar como un referente en el resurgir del movimiento nacionalista vasco en la demanda de su identidad nacional y en la lucha contra el régimen de Franco. Será éste un nacionalismo que romperá con muchos de los valores del nacionalismo tradicional.

\subsection{Casa, grupo doméstico y ámbitos de sociabilidad comunitaria}

El grupo doméstico se ha constituido en la unidad básica de la estructura social y económica de la sociedad tradicional vasca (Homobono, 1991). La casa ha sido una institución total y multifuncional, puesto que se ha constituido al propio tiempo como unidad de producción, un lugar de residencia para una familia troncal (extensa) y un signo emblemático de la cultura vasca. Hasta las primeras décadas del siglo xx, la vida en el caserío representaba una economía en gran medida autosuficiente y orientada hacia la subsistencia del grupo doméstico. Dentro del núcleo familiar extenso se desarrolla una tupida red de relaciones y ayuda mutua, de complicidades que abarcan los ámbitos productivos y de ocio.

En la sociedad tradicional vasca, las relaciones sociales que se inician en el ámbito doméstico tienen su extensión en el espacio comunitario que se genera en la vecindad, en las anteiglesias y en los barrios y pueblos. Estas relaciones, reproducen la identidad colectiva y, estructuran la vida social de los habitantes que se localizan en torno a un núcleo central donde se erige una ermita (Homobono, 1988). Entre ellos se realizan actividades y trabajos comunes, considerados de utilidad general, a escala de barrio o de pueblo: auzolan.

Lafourcade (1983) explica el carácter diferenciado del derecho consuetudinario vasco. Éste tenía un carácter colectivo y no individualista. El heredero del caserío era en realidad sólo el administrador de la hacienda familiar, dado que no la podía vender. La propiedad era de hecho de la familia. A efectos de la transmisión de la casa familiar, el sexo no era tenido en cuenta. El hijo/hija mayor era la responsable de dar continuidad al patrimonio familiar. De acuerdo con el derecho consuetudinario, para mantener los equilibrios patrimoniales en el valle, el primogénito de una familia (tanto si es hombre como si es mujer) no podía casarse con el primogénito de otra. Sobre la base familiar se construían las relaciones de gobierno municipal de carácter democrático. Así, por ejemplo, en el Biltzar de Lapurdi, en cada pueblo las asambleas de dueños de las casas se reunían a la salida de la misa del domingo. Cada casa tenía una voz, cualquiera que fuera su importancia o posesiones. Los dueńos de las casas decidían todo lo que concernía a la comunidad, siempre en función de las mayorías. Luego designaban a un representante para la Junta General, donde cada parroquia tenía una sola voz. La democracia, por lo tanto, no era basada en el individuo sino que tenía base familiar. Lafourcade afirma que «los vascos no han sido nunca indivi- 
dualistas. Se ve en su amor por la casa, por la familia, por la comunidad. Eso se encuentra a día de hoy en la proliferación de una fórmula singular de trabajar, como son las cooperativas, que es todo lo contrario al individualismo. Es una característica muy importante de los vascos, que debe perdurar» ${ }^{3}$.

En torno a los vecindarios rurales propios de una sociedad tradicional, se crearon entre los grupos domésticos, asociaciones de ayuda mutua y de carácter voluntario: la hermandad, la cofradía y el concejo. Junto a ellos, la cuadrilla de amigos y la sociedad gastronómica, extendida esta última sobre todo en Gipuzkoa, a partir de la segunda mitad del siglo XIX, han sido también importantes instituciones de socialización. Ambas, destacan por su carácter masculino e igualitario como base para el desarrollo de relaciones de amistad, orientadas al ámbito informal y de ocio.

\subsection{La Iglesia como institución social central de la vida social}

Durante los años 40 y 50, la Iglesia fue una institución social central en el País vasco, conformadora de los usos y las costumbres de la población, prácticamente la única institución que contaba con ascendencia moral sobre la gran mayoría de la población.

A lo largo del siglo xix y primeras décadas del siglo xx, la Iglesia y el carlismo compartían muchos valores de carácter tradicionalista. No obstante, la Iglesia vasca evolucionó desde posiciones ideológicas integristas hacia la defensa de la cultura y la identidad vascas ${ }^{4}$.

Tal y como afirma Itzaina (2001), el cristianismo ha contribuido poderosamente en la construcción de la identidad vasca a través de la articulación de valores, creencias y conocimientos asociada a la socialización religiosa de los actores. Barandiaran escribe al respecto, «son la fe y las esperanzas cristianas las que, en realidad, han formado el entramado de la vida del vasco durante siglos hasta nuestros días» (Garmendia, 1985: 36).

Todos estos elementos, que he ido citando de manera esquemática, han cimentado la compleja realidad cultural y simbólica de los habitantes de Mondragón y de sus aledaños a lo largo de las últimas décadas. Podemos decir con Moscovici, que, en este contexto, se ha generado una representación social esto es «una modalidad particular de conocimiento cuya función es la elaboración de los comportamientos y la comunicación entre individuos. Es un corpus organizado de conocimientos y una de las actividades psíquicas gracias a las cuales los

\footnotetext{
3 Noticias de Gipuzkoa 3 de febrero de 2012.

${ }^{4}$ En el año 1936 el párroco de Mondragón, junto con dos sacerdotes más, fueron fusilados por sus simpatías nacionalistas y por el apoyo dado a la creación de la primera ikastola de Mondragón y a la difusión de la cultura y la lengua vasca.
} 
hombres hacen inteligible la realidad física y social, se integran en un grupo o en una relación cotidiana de intercambios, liberan los poderes de su imaginación" (Moscovici, 1979: 17-18). Las propuestas de Arizmendiarrieta, creador intelectual de la Experiencia Cooperativa de Mondragón, van a ser fácilmente inteligibles para quienes han sido socializados en este contexto simbólico y cultural.

\section{La creación de un discurso moral generador de una base normativa propia}

Recordamos que el pilar normativo destaca las reglas normativas, las cuales introducen una dimensión prescriptita, evaluadora y de obligación dentro de la vida social. Incluyen tanto valores como normas, y definen metas y objetivos además de las normas apropiadas para conseguirlos (Scott, 1995).

Arizmendiarrieta ${ }^{5}$, a través de sus escritos, va a proporcionar estos valores sociales, normas morales, metas y objetivos que va a requerir el nuevo modelo socio-económico que propugna y que define en los siguientes términos:

«Consideramos que la empresa debe ser una comunidad humana de actividades e intereses, basada en la propiedad e iniciativa privada (salvo en el caso en que por causas del bien común intervenga el Estado, instituida para prestar a la sociedad un servicio de producción necesario o conveniente, mediante el cual recibe una contraprestación económica acorde con el servicio prestado, que es distribuida entre sus miembros de una manera justa.» (Arizmendiarrieta, Caja Laboral Popular, 1997: 95)

Esta es la definición de empresa que aporta Arizmendiarrieta, entendida como una aspiración de carácter moral para el logro de una comunidad de trabajo. Los elementos que configuran esta comunidad de trabajo son: la persona, la cooperación, el desarrollo comunitario y la acción pragmática. Todo ello encaminado hacia la creación de unas nuevas relaciones sociales en el ámbito del trabajo sustentadas en la justicia y la igualdad como germen para la transformación de la sociedad.

\subsection{La persona y la doctrina social de la Iglesia}

El cristianismo social es uno de los elementos que mayor influencia ejerce en el pensamiento de Arizmendiarrieta, de modo que gran parte de su reflexión intelectual está fuertemente inspirada en él. El cristianismo social de Arizmen-

5 Nacido en Markina (Bizkaia, 1915), tras realizar sus estudios en el Seminario de Vitoria y ordenarse sacerdote, fue destinado en 1941 a Mondragón, donde residió hasta su muerte (1976). En el desarrollo de su labor sacerdotal, tomó conciencia de las necesidades de su pueblo, volcándose en el desarrollo del proyecto cooperativo del que fue su creador intelectual (Molina, 2005). 
diarrieta queda sustentado sobre dos ejes de pensamiento: la Doctrina Social de la Iglesia, reflejada en las encíclicas papales, por un lado, y el Neotomismo Aristotélico de Jacques Maritain con su filosofía personalista, por otro lado (Azurmendi, 1987).

La Doctrina Social de la Iglesia es el conjunto de normas y principios referentes a la realidad social, política y económica basado en el Evangelio y en el magisterio de la Iglesia Católica. Las encíclicas papales han ido recogiendo todo ese magisterio, siendo las más importantes dentro de este ámbito las escritas por León XIII (De Rerum Novarum, 1891), Pío XI (Quadragesimo Anno, 1931) y las escritas por Juan XXIII (Mater et Magistra y Pacem in Terris, 1961 y 19636). Todas ellas centran su atención en las condiciones de vida de los trabajadores, la búsqueda de la justicia social, rechazan las teorías marxistas, definen el lugar de la propiedad en la sociedad que, sin negar su carácter de "derecho natural», promueva su difusión entre todas las clases sociales y proponen superar las desigualdades subrayando el papel de las personas como sujeto y objeto del bienestar. Todas estas ideas ejercerán una gran influencia en el pensamiento de Arizmendiarrieta y se van a constituir en su guía de acción. Concretamente, la Encíclica Quadragesimo Anno alienta las vías cooperativas para las empresas.

A su vez, Arizmendiarrieta observa cómo a partir de los años 50, de manera más destacada, las ideologías de izquierda (socialista y comunista) comienzan a tener un prestigio cada vez más extendido entre las masas obreras, apreciando cierto alejamiento de éstas respecto de la Iglesia. Este hecho lo atribuye a que la Iglesia está alejada de las preocupaciones y necesidades de los mismos. Siendo esto así, estima necesario un cambio en el mensaje y en la acción de quienes forman parte de la Iglesia. Arizmendiarrieta encuentra en las Encíclicas Papales el mensaje y la legitimación que él necesita para realizar la labor que está impulsando en respuesta a las necesidades de la población. Su actividad social, la desarrollará, tal y como estableció Pío XI a través de la Acción Católica ${ }^{7}$, que de hecho era la única institución admitida por el régimen para desarrollar actividades de índole social o económica.

La segunda gran influencia es la aportada por el Neotomismo Aristotélico, corriente filosófica impulsada por Jacques Maritain («Humanismo Integral»,

${ }^{6}$ La publicación de las dos últimas encíclicas coincide en el tiempo con la efervescencia del movimiento cooperativista de Mondragón y de otras zonas del País Vasco.

${ }^{7}$ La Acción Católica fue fundada en 1920 por el prelado belga Joseph Cardijn quien inició su labor pastoral entre los jóvenes obreros belgas. Agrupó a los jóvenes obreros de Bruselas en la llamada juventud sindicalista (1919), que se convertiría posteriormente (1924), en la Juventud Obrera Católica (JOC). Por medio de estas organizaciones trató de llevar a la práctica el compromiso social de la Iglesia. No obstante, a la Acción Católica Española, bajo la dirección de Mons. Zacarías Vizcarra le será encomendada por Franco la tarea de recristianizar la sociedad y restaurar el país a tono con una "cristiandad victoriosa» (Urbina, 1977: 19-21). «Arizmendiarrieta ha mostrado una visión bien distinta de las funciones de la Acción Católica» (Azurmendi, 1992: 188). 
1966), junto a Mounier, Nedoncelle, Marcel y Guardini, dando lugar al pensamiento personalista ${ }^{8}$. Maritain, junto con Mounier, destaca el protagonismo del hombre en su tarea de construir y habitar el mundo con otros. El mundo a su vez vuelve a actuar sobre la persona, y así de manera sucesiva, de modo que en la relación entre la naturaleza y el mundo social la propia persona se transforma, transformando. De este modo, el hombre hace historia, porque la historia consiste en ese flujo de fuerzas donde el hombre es el actor principal. El personalismo comunitario afirma que la persona es el valor fundamental, un modo de vida que sitúa a la persona en el centro de sus reflexiones y sus acciones. Sus reflexiones sobre la persona y la existencia comunitaria, están orientadas a formar un criterio que guíe la acción para hacer posibles unas determinadas estructuras políticas, económicas, sociales y culturales al servicio de la persona. Se trata de un pensamiento que llama al compromiso. Estas ideas del personalismo van a ser asumidas por Arizmendiarrieta y van a constituir un elemento legitimador relevante para la creación de las cooperativas, constituyendo el eje central y la fuerza motriz para su proyecto social comunitario y personalista?

${ }^{8}$ Maritain, Mounier y el movimiento personalista francés eran conocidos en Euskadi ya antes de la guerra. El movimiento social vasco cristiano, los tenía en gran estima. Sin embargo el renombre de estos autores alcanzará su culmen cuando, en la guerra civil, denostados por su propia jerarquía eclesiástica, calumniados, escarnecidos y excomulgados por la jerarquía española, despreciados por el Vaticano, los nacionalistas vascos encuentren en ellos los únicos valedores de su causa en un mundo católico enteramente favorable a Franco.(...) Maritain formará parte del Comité de ayuda a los exiliados vascos, como vicepresidente de la «Ligue Internationale des Amis des Basques» (Azurmendi, 1992: 110).

9 Por otra parte y de acuerdo con Race Mathews (1999), el pensamiento de Arizmendiarrieta puede ser entendido como un «distribuismo evolucionado». El distribuismo en su forma original fue desarrollado por el escritor inglés Hilaire Belloc, quien trató de dar un enfoque práctico a las enseñanzas de De Rerum Novarum. Su fundamento es la creencia de que sólo se puede alcanzar un orden social más justo por medio de una distribución de la propiedad mucho más extensa. El distribuismo favorece una «sociedad de propietarios» donde la propiedad pertenece a muchos en lugar de a unos pocos y se opone a la concentración de la propiedad en manos de los ricos, como en el capitalismo, o del estado, según lo propugnado por la ideología marxista. Específicamente, la propiedad de los medios de producción, distribución e intercambio debe ser difundida. La plataforma de la Liga Distribuista, que Belloc y G.K. Chesterton fundaron, indicaba abiertamente que «cada trabajador debería poseer una parte de los activos y el control del negocio en el cual trabaja» (Mathews, 1999). Los distribuístas también favorecieron fuertemente las cooperativas y tuvo un gran arraigo en Nueva Escocia (Canadá), con el Movimiento de Antigonish, que los sacerdotes católicos Moses Coady y Jimmy Tompkins establecieron en los años 20. Los Papas Pío XI, y Pío XII apoyaron firmemente este movimiento e incluso este último nombró a Coady Prelado nacional con rango de Monseñor. Tal nombramiento fue realizado el año 1946, cuando Arizmendiarrieta estaba realizando sus trabajos en torno a la Escuela Profesional. Parece cuando menos extraño que Arizmendiarrieta no tuviera conocimiento del mismo. Estuviera al corriente o no, lo cierto es que existe un claro paralelismo entre en Movimiento Antigonish y el pensamiento de Arizmendiarrieta en lo referente al papel del trabajo y de la propiedad y la doctrina del subsidiario (una entidad superior no debería asumir funciones en nombre de una identidad inferior que es capaz de llevarlas a cabo por sí mismo). Para ambos movimientos, son los trabajadores quienes deberían contratar el capital y no al contrario, de tal modo que se les permita asumir a los trabajadores la propiedad de sus lugares de trabajo y convertirse en dueños de sus propios destinos. 


\subsection{La cooperación}

Una segunda idea o principio en el pensamiento de Arizmendiarrieta es la idea de cooperación que en 1948 describe en los siguientes términos:

«Para mí es la clave y hasta, si queréis, el secreto atómico, llamado a revolucionar toda la vida social. Colaboración de clases, colaboración del pueblo con sus autoridades y de las autoridades con el pueblo, colaboración de la teórica y del espíritu es el secreto de la verdadera vida social y la clave de la paz social.» (Arizmendiarrieta, Escuela Profesional, 1997: 68-69)

La posición de Arizmendiarrieta en torno a la cooperación está perfectamente alineada con la posición de la Iglesia Católica anteriormente expuesta y opuesta a la concepción marxista de la lucha de clases ${ }^{10}$. Por otra parte, la idea de cooperación está vinculada íntimamente a las ideas de solidaridad, espíritu de superación y sentido del trabajo.

La solidaridad cooperativa se manifiesta a varios niveles. Un primer nivel es la solidaridad intracooperativa, que se evidencia en la actitud de compromiso de los trabajadores con su proyecto de empresa comunitaria y que se refleja en aspectos tales como regulación de los índices salariales. La solidaridad, en segundo lugar, se manifiesta también en las relaciones entre cooperativas, de manera que trabajen y se coordinen conjuntamente. Este aspecto quedará bien reflejado dentro del cooperativismo de Mondragón con la creación de organismos de cohesión y reglas de funcionamiento que hagan realidad dicha solidaridad. El tercer nivel de solidaridad es el comunitario, de modo que las organizaciones cooperativas han de volcarse en la comunidad de la que han surgido y que es la razón de ser de las mismas, de modo que genere riqueza y recursos para la comunidad a la que está unida.

El espíritu de superación y el sentido del trabajo están también íntimamente vinculados a la cooperación. La cooperación está unida a la necesidad de superar dificultades, a la necesidad de unir a las personas para la resolución de problemas o la generación de recursos, "siempre hay un paso más que dar» nos dice Arizmendiarrieta (Caja Laboral Popular, 1997: 249).

El trabajo, por su parte, es otro de sus conceptos nucleares y al igual que muchos de los conceptos que utiliza, adquiere un carácter polisémico. El tra-

10 Ideología marxista que, a finales de los años 60 y comienzos de los 70, va a ejercer su influencia entre sectores del nacionalismo vasco y del movimiento sindical y va a formular una crítica frontal al proyecto cooperativo y a las tesis de Arizmendiarrieta. Estos sectores van a considerar el proyecto de Arizmendiarrieta como una iniciativa que rompía la unidad de la clase obrera y por tanto la debilitaba en su lucha contra el régimen franquista y en defensa de la identidad nacional y social del Pueblo Vasco. A finales de los 60 y durante los años 70, años de gran actividad política y movilización social en contra del franquismo, las cooperativas, recibieron fuertes críticas debido a que no se sumaban a las huelgas que convocaba el incipiente movimiento sindical y político de la época (Kasmir, 1999). 
bajo está vinculado a la autorrealización del hombre, («es lo más grande que un hombre puede dar» (Azurmendi, 1992: 268)); y a la propiedad de sus frutos (la propiedad no estará mejor que en manos de quienes trabajan). Es también la manera de ayudar a Dios a transformar la naturaleza para la promoción del ser humano. Tiene, por tanto, un sentido práctico y al mismo tiempo trascendente. El trabajo le daba al individuo no solamente su sustento sino también su identidad, autoestima y capacidad de participar en una sociedad civil por completo.

La idea de la cooperación para que pueda ser desplegada y dé lugar a los resultados que de ella se espera requiere de una serie de presupuestos que es preciso asegurar. Estos presupuestos son: la educación, la comunidad sensibilizada, el espíritu comunitario y los elementos humanos y económicos.

La educación ocupa un lugar central en la reflexión que realiza Arizmendiarrieta: «saber es poder y para democratizar el poder hay que socializar previamente el saber» (Arizmendiarrieta, Escuela Profesional, 1997: 335-336). Por tanto, la mejor inversión que puede realizar un pueblo es la formación de sus miembros. La educación tal y como él la entiende no consiste únicamente en la adquisición de una serie de técnicas, que aun siendo importante no es suficiente, dado que la educación ha de ser la búsqueda de un sentido a la vida. En su caso, ese sentido viene dada por la cooperación, de modo que la educación y la cooperación quedan unidas en su proyecto, ambos se complementan mutuamente.

Para que la cooperación se despliegue en toda su potencialidad es necesaria una comunidad sensibilizada y socialmente concienciada. La cooperación surge de la comunidad, es en ella en la que se asienta y a la que sirve, siendo también la comunidad su razón de ser y su fin último. La conciencia social de la comunidad tiene diferentes facetas o manifestaciones: una conciencia de libertad que requiere su encarnación en realidades económico-sociales; una conciencia de justicia social, inductora de un nuevo régimen de solidaridad y orden social; una conciencia de desarrollo, con la exigencia de la movilización de recursos potenciales, trabajo y ahorro y, por último, una conciencia participativa que requiere de un control y una acción democrática.

Junto a lo anterior, la cooperación necesita de la existencia de espíritu comunitario. «El cooperativismo centra más su atención en la comunidad que en el individuo» (Arizmendiarrieta: Formación Cooperativa, 1997: 95), de hecho concibe la cooperativa como una comunidad de trabajo donde lo que prima es el interés comunitario.

Por último, son necesarios los recursos económicos y humanos. Sin ellos, cualquier proyecto, por pequeño que sea, es imposible de materializar. El proyecto cooperativo, ambicioso e ilusionante, requiere de recursos económicos y de personas preparadas técnicamente y humanamente para llevarlo a la práctica. 
"No se nos ocurra hacer cooperativas sin cooperativistas: es más, los cooperativistas con los que queremos hacer cooperativismo necesitan ser hombres capaces y competentes en los diversos dominios de la técnica o de la profesión, acatando sus exigencias. A todo ello han de añadir una fina sensibilidad social y una capacidad de convivencia y relación bajo los imperativos de la solidaridad.» (Arizmendiarrieta, Formación Cooperativa, 1997: 159)

\subsection{Desarrollo comunitario}

La acción cooperativa impulsada por Arizmendiarrieta está encaminada a lograr la promoción humana y social de la persona. Esta promoción, tal y como lo entiende Arizmendiarrieta, en el contexto de subdesarrollo en la que estaba sumida la población en la década de los 40 y 50, requiere como condición primera del desarrollo económico, para poder avanzar más adelante en un mayor bienestar social, cultural y humano. Este desarrollo económico, para que sea realmente transformador, ha de ser comunitario. Es la comunidad en su conjunto el sujeto del progreso económico para que toda ella pueda promocionarse social, cultural y humanamente. La persona sólo podrá aspirar a mejorar social y económicamente en la medida en que esté inmerso en un proceso comunitario de desarrollo. Este planteamiento muestra claras connotaciones con el pensamiento personalista.

La cooperativa es la palanca o herramienta del cambio social. Arizmendiarrieta percibe claramente el papel de lo económico dentro de la sociedad, siendo la empresa el máximo exponente del mismo. Es por ello, que la transformación de la empresa es el primer paso para la transformación de la propia sociedad.

Por otra parte, siendo el desarrollo económico de la empresa una condición para lograr un desarrollo de carácter «integral», es preciso poner todos los medios técnicos, organizativos, materiales y humanos para asegurar dicho desarrollo económico. La empresa cooperativa ha de demostrar su viabilidad económica en un contexto dominado por las empresas capitalistas, haciendo uso de todas las capacidades e innovaciones a su alcance pero sin perder de vista cuál es el espíritu que lo anima.

\subsection{Acción desde el pragmatismo}

Arizmendiarrieta «es un hombre de acción» (Perez de Calleja, 1989), concibe la vida como lucha: «vivir es luchar, queramos o no queramos. Porque hay que luchar para saber, hay que luchar para poder, hay que luchar para querer, hay que luchar para desarrollar las facultades, para ser algo" (Arizmendiarrieta, Primeras Realizaciones, 1997: 121). La vida en Arizmendiarrieta tiene un sentido de sacrificio y disciplina, de renuncia al hedonismo y al camino fácil, de honda raíz cristiana.

La acción y la lucha son los síntomas de vitalidad de un colectivo. Es por ello que todo su pensamiento y todas sus aportaciones están dirigidos a movili- 
zar las conciencias para la consecución de un nuevo orden social. Se podrán dar pasos sucesivos en esa dirección en la medida en que el hombre vaya tomando conciencia de la situación en la que vive. De modo que a cada nivel de conciencia histórica corresponde un tipo de acción, siendo ésta de carácter dinámico y progresivo. Por ello, las cooperativas no son más que una experiencia,

«El cooperativismo no debemos vivirlo como si lo aceptado y dispuesto en un momento fuera algo invariable sino más bien admitiéndolo como un proceso de experiencia en el que pudieran y tuvieran que adoptarse cuantas modificaciones contribuyeran a, dejando a salvo la nobleza y categoría de los altos fines perseguidos, a la actualización de los medios.» (Arizmendiarrieta, Formación Cooperativa, 1997: 181-182)

El nuevo orden al que aspira Arizmendiarrieta y hacia el que quiere ir dando pasos es un orden dinámico:

«El orden hacia el que tiende el cooperativismo no es estático, es un orden en permanente evolución hacia una forma mejor, es un equilibrio en movimiento. Una acción inerte es una contradicción y el cooperativismo que ha nacido de la acción y de la experiencia más que de la teoría es algo que debemos concebirlo y desearlo en la búsqueda de mejores formas de expresión.» (Arizmendiarrieta, Formación Cooperativa, 1997: 10)

El cooperativismo de Arizmendiarrieta está sujeto a cambio y a transformación y el cambio es concebido como algo natural, siempre al servicio de un futuro más humano:

"Si el signo de vitalidad, en definitiva, no es durar sino renacer, como dijo muy bien un gran cooperativista, si el cooperativismo no es sólo la antípoda del paternalismo sino también del conformismo y del conservadurismo, y no está atado a ningún dogmatismo, se impone que estemos en la vanguardia de las innovaciones sociales, máxime cuando éstas están demandadas por una conciencia de dignidad y de libertad, de justicia y de solidaridad.» (Arizmendiarrieta, Caja Laboral Popular, 1997: 45)

Por último, es preciso remarcar que las ideas de Arizmendiarrieta en torno al cooperativismo están dispersas en infinidad de artículos, editoriales de la revista "Trabajo y Unión», sermones, conferencias, etc. No se trata pues de un pensamiento elaborado a modo de ensayo literario concluyente y definitivo. Su pensamiento, evoluciona de acuerdo con los acontecimientos y con sus experiencias, sin sujetarse a excesivos dogmas más allá de las ideas anteriormente expuestas. Esta realidad que apela a su conciencia, es la condición miserable del hombre tanto en lo material como en lo moral. Ese es el punto de partida de sus reflexiones, pero éstas sólo tendrán validez en la medida en que aportan soluciones o pueden ser puestas en práctica. De lo contrario, serán desestimadas: 
"Doctrina que no se pone en obra, convicciones que no se traducen en actos son algo tan anormal como vida que no late. No estamos puestos en el mundo para contemplar o lamentar, sino para transformar.» (Arizmendiarrieta, Sermones, 1997: 252)

\section{Las bases reguladoras e innovadoras al servicio del proyecto socio-empresarial}

En tercer lugar, la ECM, en su proceso de institucionalización, requiere de un pilar regulador responsable de las presiones coercitivas, reglas y normas que promueven ciertos tipos de comportamientos a la vez que prohíben otros. Este pilar está sancionado legalmente y esta es su fuente de legitimidad. Las instituciones establecen una serie de reglas y, a través de mecanismos tanto formales como informales, son capaces de regular las actividades de las organizaciones.

Estas bases reguladoras fueron desde el comienzo una gran preocupación para Arizmendiarrieta. No olvidemos que desde que se inicia la primera actividad empresarial en Vitoria en noviembre de 1955, bajo la forma jurídica de sociedad anónima, hasta abril de 1959, en la que adopta un encuadre jurídico de sociedad cooperativa, transcurren casi cuatro ańos. En ese periodo, Arizmendiarrieta analizó las diferentes formas jurídicas existentes, tratando de definir la más adecuada para su proyecto socio-empresarial. Finalmente elaboró unos estatutos bajo el marco cooperativo, pero con un carácter heterodoxo que dificultaba su encaje en el marco jurídico existente, pero que finalmente encontrará acomodo dentro de la Ley de Cooperativas del año 1942.

\subsection{Reglas relativas al tratamiento del capital}

La figura del socio de la cooperativa fue hasta cierto punto mitificada en el imaginario de Mondragón para remarcar la responsabilidad que asumían los integrantes del cooperativismo. En este sentido, se caracterizaba al socio en contraposición al «hombre corriente», en los siguientes términos:

«Los primeros [los hombres corrientes] preferirán la comodidad del lamento y los segundos [los socios] buscarán la Responsabilidad de la tarea asumida. Estos tienen en la cooperativa su marco jurídico-social idóneo para, junto con los demás socios, buscar la consecución de una empresa económicamente rentable y socialmente enriquecedora. Estos quieren dejar la huella en la historia grande o pequeña de la sociedad en la que viven. Son los capaces de analizar y sintetizar los acontecimientos y, sobre todo, los que apuestan por la mejora individual y social. Si hay una característica que destaque en el primero es el pesimismo y, por el contrario, el rasgo más notable del segundo es el optimismo.» (Larrañaga, 1984: 10)

En esta manera de caracterizar al socio, se observan claras reminiscencias del modo en que Arizmendiarrieta veía al hombre de empresa, «el gran capitán de 
empresa» que él tanto admiraba: la persona emprendedora, que asume riesgos, que se esfuerza por la consecución de un fin y es capaz de asumir el papel de liderazgo de un grupo para encaminarlo hacia el objetivo. No es correcto, por lo tanto, identificar su idea de empresario con la de capitalista.

Desde un punto de vista empresarial y pragmático, «la palabra socio, tiene connotaciones de propiedad, de jefe y de patrón. El socio, junto a otros iguales a él, participa en los beneficios y en las decisiones, lo que le eleva a los niveles privilegiados de la sociedad» (Larrañaga, 1984: 9).

Para ser acreedor de la condición de socio es preciso cumplir necesariamente dos requisitos básicos: aportación de trabajo y aportación de capital.

La primera supone identificar al socio como agente productivo, como generador de riqueza y como transformador de la naturaleza. La segunda, supone identificar al socio trabajador como copropietario de los medios de producción y acreedor a las responsabilidades de dueño, junto a sus compañeros de empresa.

En relación al tratamiento del capital en los estatutos de ULGOR, los aspectos considerados poco ortodoxos de acuerdo con la legislación vigente (Ley de cooperativas de 1942) son los siguientes (Larrañaga, 1981: 137):

—Considerar el interés al capital, como «salario» del capital, en paridad con el salario o anticipo del trabajo.

- Remunerar en forma variable al capital, haciéndolo acreedor al «retorno» en igualdad de condiciones que al trabajo, en lógica con el reconocimiento del anticipo al capital en su interés.

- Incorporar la noción de prima de riesgo, como remuneración adicional al capital, por la especial naturaleza fungible, en caso de quiebra o pérdida.

Estas disposiciones obedecen al deseo de proporcionar las mejores condiciones al capital para atraerlo a las cooperativas, dadas las dificultades inherentes al cooperativismo para lograr el capital suficiente que sustente la actividad empresarial. Con este objetivo, incluso es admitido dentro de las cooperativas el denominado capital comanditario, esto es aportaciones de capital por parte de personas que no van a ser trabajadoras de la cooperativa. Estos aspectos eran del todo «inusuales» en las cooperativas, no obstante, son finalmente admitidos. Por tanto, para adquirir la condición de socio, se debe de realizar una aportación de capital de carácter obligatorio, igual para todos ellos (base igualitaria del cooperativismo). Este capital aportado recibirá su correspondiente remuneración dado que «el capital es el trabajo excedente o el patrimonio social destinado para instrumentar adecuadamente la actividad presente y su prestación será acreedora a la justa remuneración que le corresponda en 
una equilibrada compensación de sacrificios pasados y presentes» ${ }^{11}$. Además, se establecerá una remuneración adicional al capital en compensación al riesgo («independientemente a la retribución variable complementaria a todo capital retenido, voluntario y comanditario en compensación al riesgo que entrañaba la inversión realizada» ${ }^{12}$ ).

Las cantidades que los socios, en los años cincuenta, aportaban para incorporarse a las cooperativas eran en torno a las 50.000 pesetas (300 euros), en algunos empresas llegaba incluso a las 75.000 pesetas (450 euros), cantidad muy elevada para la época. Los trabajadores se veían en dificultades para sumar esas cantidades si bien se daban facilidades y plazos para realizar el desembolso. En muchas ocasiones, los trabajadores recurrían a familiares o conocidos para reunir dicho capital. Entre ellos se encontraban con frecuencia los baserritarras que en los ańos cincuenta dispusieron de cierta capacidad económica por encima de la media de la población. Ello era debido a los beneficios económicos que obtuvieron por sus explotaciones forestales, concretamente con la explotación del pino insignis, que se vendía a las empresas papeleras del entorno, las cuales realizaban una gran demanda. Mucho de este capital fue a parar a las cooperativas, bien a modo de aportación económica por parte de baserritarras que abandonaban el caserío como explotación agropecuaria pasando a trabajar en las cooperativas en condición de socio, o bien a través de la apertura de cuentas corrientes en las oficinas de la incipiente Caja Laboral Popular, fundada en enero de 1960. Muchos baserritarras incluso, hacían la aportación de capital con la promesa de incorporarse con posterioridad a la cooperativa, cuando hubiera necesidad de mano de obra, poniendo de manifiesto la confianza que primaba en las relaciones. De esta manera, el problema de lograr capital, problema endémico de las cooperativas, estaba en camino de ser encauzado, dado que se había logrado una fuente de financiación colectiva.

Los niveles salariales establecidos se situaron en una banda salarial de uno a tres, lo cual contribuyó a una mayor cohesión interna y una mayor solidaridad entre los miembros de la cooperativa, dado que tenían un carácter igualitario. Así, el artículo 7 de los estatutos de ULGOR establecía que

«Al objeto de conjugar las exigencias de una vinculación equitativa y mutua con las del rendimiento efectivo y el estímulo de superación de todos los componentes de la entidad, se adopta, para el ejercicio de los derechos sociales y la percepción de los anticipos laborales, una escala de valoración que abarca a todos los asociados y cuyos índices extremos serán de uno y tres.» (Riaza, 1968: 119)

11 Artículo 6 de los Estatutos de ULGOR, citado por Larrańaga (1981: 140-141).

12 Artículo 49 del reglamento de régimen Interior de ULGOR, citado por Larrañaga (1981: 141). 
Este planteamiento quedaba corroborado por el artículo 8, donde se recogía que

«Para estimular y coordinar la máxima cooperación personal de los socios a los fines de la Cooperativa se procederá a una clasificación profesional de los mismos, en el momento de su incorporación a la Sociedad, con arreglo a su aptitud, que periódicamente será renovada en consonancia con su actividad, y los anticipos laborales serán proporcionales a su categoría profesional y eficacia.» (Riaza, 1968: 119)

Los excedentes económicos (beneficios), se capitalizan. No obstante, los excedentes obtenidos en uno de los años iniciales, se monetarizaron, lo cual ayudó a elevar aun más el ánimo; se trabaja intensamente, pero la satisfacción es grande dado que el contexto socio-económico es favorable. El hecho de obtener unos resultados económicos sorprendentemente favorables desde el inicio, contribuyó muy positivamente a superar la desconfianza y los recelos que existían en torno al funcionamiento de las cooperativas. La superación de estas dificultades, les insufló de confianza y de legitimidad dentro de la comunidad.

En relación al reparto de excedentes económicos ${ }^{13}$, obedecía a los siguientes criterios:

— 10\% al Fondo de Educación y promoción Social.

- 45\% al Fondo de Reserva, como mínimo.

$-45 \%$ se reparten a los socios (son capitalizados) a modo de retorno.

Subrayamos el hecho de que menos de la mitad de los resultados positivos obtenidos se repartían entre los socios ${ }^{14}$, si bien se capitalizaban. Siendo ello un síntoma del compromiso y de la responsabilidad de los trabajadores para con su empresa.

Por otra parte, hay que señalar que el reparto de excedentes entre los socios se realizaba de manera proporcional a las actividades, servicios u operaciones realizadas con la cooperativa, que estaba reflejado en su índice laboral. Si bien en aquella época las ganancias fueron abundantes y continuadas, cuando las cooperativas tienen pérdidas (cosa que ocurrió a finales de los ańos setenta),

13 En el lenguaje cooperativo se utiliza el término excedentes económicos en lugar de beneficios, término éste que se considera no del todo coherente con la fundamentación ideológica del cooperativismo. No obstante, la Experiencia Cooperativa Mondragón, desde su inicio, fue ajena a esta reflexión.

14 En al actualidad y de acuerdo con la Ley Vasca de Cooperativas de 1993, la distribución de excedentes se realiza de la siguiente manera: $20 \%$ al Fondo de Reserva Obligatoria; $10 \%$ al Fondo de Educación y Promoción Cooperativa; el resto lo distribuirá la Asamblea General entre los destinos siguientes: 1) Retorno a los socios y participación de los asalariados sin opción a ser socios; 2) Dotación fondos de reserva voluntarios repartibles o irrepartibles. 
éstas se sufragaron mediante el capital acumulado por los socios, realizando los extornos o retornos negativos correspondientes.

El capital que los socios van acumulando a lo largo de los años, si éstos son económicamente favorables, tal y como lo fueron de manera ininterrumpida durante los ańos iniciales de la Experiencia Cooperativa, alcanzaban sumas muy significativas. Dicho capital no podía ser monetarizado por los socios, esto es no lo podía retirar de la cooperativa hasta que causara baja en la misma, esto es normalmente, hasta su jubilación. No obstante, el socio recibe los intereses correspondientes a las sumas acumuladas, dado que su capital está sujeto a un interés similar o en ocasiones superior al interés establecido por el mercado bursátil.

De esta manera, se logró que la empresa no se descapitalizase y tuviera posibilidades de inversión $\mathrm{y}$, al mismo tiempo, gracias a los intereses pagados a su capital, que los socios lograran incrementar sus ingresos, si bien de manera diferida. Este tratamiento del capital contribuyó poderosamente a hacer de las cooperativas entidades financieramente fuertes y una mayor adhesión de los socios hacia la cooperativa.

Señalar, por último, que este tratamiento del capital acentúa la importancia del trabajador como socio capitalista de la cooperativa y consecuentemente como propietario de la misma. Se era propietario en la medida en que se aportaba trabajo y capital, pero la aportación de capital quedaba destacada desde un punto de vista tanto práctico como simbólico. Las aportaciones de capital tenían una importancia de carácter práctico indudable, en la medida en la que era la fuente de acumulación de capital necesario y extremadamente escaso. Pero, al mismo tiempo, tenía una importancia de carácter simbólico de cara a la institucionalización de la cooperativa en clave igualitaria, además de propiciar el compromiso y la identificación de sus miembros. Arizmendiarrieta elevó a los trabajadores a la condición de propietario y este hecho fue bien interiorizado por los la población dado que conectaba con su universo cultural.

\subsection{Reglas relativas al gobierno}

Un segundo aspecto en el que Arizmendiarrieta va a introducir modificaciones con respecto a la Ley de Cooperativas vigente, es el referido a la participación y a la democracia.

En Arizmendiarrieta, la democracia es consustancial a la cooperativa:

«La referencia a la comunidad como la apelación al régimen democrático, son elementos sustantivos de toda acción y filosofía cooperativista; tanto uno como lo otro presuponen naturalmente un elevado sentido de libertad y de justicia.» (Trabajo y Unión, 1968, n. ${ }^{\circ}$ 92) 
No obstante, es preciso clarificar el sentido que la democracia tiene para Arizmendiarrieta. En los estatutos de ULGOR, al referirse al gobierno democrático de la cooperativa, comience hablando de autoridad y sólo después se aluda a la designación democrática de los que la vayan a ejercer. Así establece el artículo 6 de sus estatutos:

«El trabajo como actividad racional debe ser disciplinado y el orden implica una autoridad que los socios de esta Cooperativa reconocerán en los que hayan sido designados para ejercerla, acatándola siempre rigurosamente.» (Riaza, 1968: 118)

Arizmendiarrieta entiende la democracia cooperativa en los siguientes términos:

«Es un recurso de selección de los mejores para el gobierno propio y un imperativo de acatamiento espontáneo y riguroso de las órdenes de los hombres de mando, quienes deberán ofrecer una gestión eficaz en un proceso dinámico de adaptación a las circunstancias y de superación de dificultades en provecho común. Los socios tienen el derecho de escoger para su gobierno a los hombres de su confianza y agrado con el deber, igualmente inexorable de obedecerles el periodo de su mandato sobreponiéndose a los vaivenes de su arbitrio. Los designados para el mando pueden no aceptarlo, pero una vez comprometidos con el cargo deben saber que su ejercicio no es para utilidad y provecho propio, sino en servicio a la comunidad. Ya que en este contexto mandar no es nada grato, es natural que quienes ejerzan las prerrogativas de la autoridad requieran de los demás una amplia colaboración, que es algo más que simple resignación al propio deber». Más adelante, afirma «la democracia ha de ser un método y procedimiento para escoger mejor a quienes pudieran tener aptitudes de gestión. Puede llegarse a ello por designación directa, o a través de quienes reciban facultades para ello». Y concluye, «los subordinados, una vez ejercido su derecho de designar sus autoridades, han de actuar por un lado ejecutando lo que se les ordena y por otro recurriendo a la crítica oportuna y constructiva y mediante la misma al control de la gestión encomendada a los primeros.» (Trabajo y Unión, 1963, n. $\left.{ }^{\circ} 35\right)$

El artículo 8 de los estatutos de ULGOR elaborados por Arizmendiarrieta establece que

"La democracia cooperativa es un recurso de selección de los mejores para el gobierno propio y un imperativo del acatamiento espontáneo y riguroso a las órdenes de los hombres de mando, quienes deberán ofrecer una gestión eficaz en un proceso dinámico de adaptación a las circunstancias y de superación de dificultades en provecho común.» (Larrańaga, 1981: 140)

Arizmendiarrieta tiene una gran preocupación por llevar a cabo una gestión empresarial eficaz dentro de las cooperativas y una desconfianza marcada hacia las masas y en general hacia el proletariado, éste, en su opinión, tiene poca conciencia y está poco preparado. Antxon Pérez de Calleja, Directivo de Caja La- 
boral, bajo el título "Arizmendiarrieta, un hombre de acción», nos aporta la siguiente apreciación:

"Arizmendiarrieta conservaría para siempre un delicado rechazo hacia una masa anónima a la que había visto perder con enorme facilidad la cabeza y la compostura. No ya la histeria colectiva, siquiera el entusiasmo le eran sospechosos. De ahí su predilección por el trabajo en "petit comité", su elección deliberada y consciente de la élite como auténtica protagonista de la historia y su papel en ella como formador de hombres, en el cara a cara, en la implicación personal, en el gusto por la confidencia y el trato directo.» (Pérez de Calleja, 1989: 14-15)

Arizmendiarrieta se dedicará a formar a una pequeña élite que cuando las circunstancias lo posibiliten crearán las empresas cooperativas. Los directivos de las cooperativas van a ser seleccionados con esmero, tanto por sus virtudes como hombres de empresa como por su integridad moral. Una vez realizada esta selección y en la medida en que el directivo demuestra su pericia empresarial en la dirección, los trabajadores deberán de colaborar, dándoles facilidades para que pueda desarrollar su labor. Allí donde prima la confianza, la democracia como sistema que garantiza unos supuestos derechos no tiene demasiado sentido, solo sirve para ralentizar la toma de decisiones. Por tanto, la actitud requerida por parte de los trabajadores en la cooperativa no es tanto de subordinación como de disciplina.

«Entre las normas cooperativas se encuentra consignado expresamente que la democracia cooperativa es un recurso de selección de los mejores para el gobierno propio. Quienes deberán ofrecer una gestión eficaz en un proceso dinámico de adaptación a las circunstancias y de superación de dificultades en provecho común. Supuesto ello no hay razón para que cada uno por su parte no colabore y bajo esta colaboración deberemos entrańar la adopción de una actitud de comprensión con los que hubiéramos elegido para que la correspondencia no exija heroísmos a los mismos", y concluye: "por vía de la comunicación se debe poder mantener la corresponsabilidad articulada en todos los niveles y con ella se debe tratar de hacer fecunda la gestión y llevadera la tarea directiva.» (Trabajo y Unión, 1970, n. ${ }^{\circ} 122$ )

No obstante, los trabajadores tienen derecho a ejercer el control democrático de la gestión realizada, «los trabajadores, una vez ejercido su derecho de designar sus autoridades, han de actuar por un lado ejecutando lo que se les ordena y, por otro, recurriendo a la crítica oportuna y constructiva y mediante la misma al control de la gestión encomendada a los primeros. Por tanto la información y la transparencia en la gestión deben de estar garantizadas».

Es significativo señalar que en los primeros estatutos de ULGOR, el derecho al voto fue cualitativo, es decir que cada persona tenía tantos votos como su índice laboral. Dado que los índices laborales recorrían una escala de 1 a 3 , el valor del voto era ponderado en la misma proporción: 
«Los socios adoptarán la disciplina, la jerarquía y las compensaciones derivadas de su propia clasificación que será consignada en coeficientes unitarios según valoración de los respectivos puestos de trabajo, en escala de índices extremos uno y tres. Estos índices serán los módulos para el ejercicio de los derechos sociales y económicos, respectivamente.» (artículo 4 de los estatutos de ULGOR)

En opinión de Jesús Larrañaga, este voto cualitativo «refleja el trasfondo de duda sobre actitudes colectivas en materias económicas, y la conveniencia de proteger, por supuesta correlación de capacidad profesional y sensatez opinática, a la empresa de frivolidades colectivas» (Larrañaga, 1981: 140). Esta norma estuvo en vigor aproximadamente durante diez años, al cabo de los cuales se apreció «que no gozaba de prestigio el sistema, era difícil explicarlo, causaba algunas aversiones y, después de todo, no modificaba lo más mínimo los resultados que se hubieren obtenido de haber ejercido su derecho todos los socios mediante un voto, según los principios elementales del cooperativismo» (Ormaechea, 1991: 6).

\section{Conclusión}

Para concluir, recordemos que este trabajo ha intentado explicar los atributos institucionales que han singularizado a la Experiencia Cooperativa Mondragón (ECM) desde su origen y los aspectos institucionales más notables que la forman. A tal fin, ha estudiado los pilares sobre los que se sostiene la ECM en su inicio y que le van a legitimar durante sus primeras décadas de existencia. Estas bases, normativas, reguladoras y cognitivas le proporcionan continuidad y significación a lo largo de los años. De este modo, tanto las instituciones como la ECM, se constituyen en pautas culturales que ofrecen recursos y valores compartidos a las cooperativas y a las actividades particulares insertándolas en macro-proyectos. Son unos sistemas simbólicos y formas de ordenar la realidad y de proporcionar una experiencia común.

A ese propósito, conviene resaltar la coherencia existente entre los tres pilares que hemos señalado como elementos integrantes del proceso de institucionalización de la Experiencia Cooperativa Mondragón. Los aspectos simbólicoculturales tienen su prolongación en el discurso moral y su reflejo en el aspecto regulativo, hasta lograr una idea de totalidad integrada. Esta concepción cooperativa es puesta en marcha y contrastada con la realidad en la medida en que se constituye como una construcción social.

\section{Bibliografía}

Aranzadi, D. (1976): Cooperativismo industrial como sistema, empresa y experiencia. Universidad de Deusto, Bilbao. 
Arizmendiarrieta, J.M.a "Caja Laboral Popular, (C.L.P.)» (1997): Textos recopilados en Pensamiento de D. José María Arizmendiarrieta CD.

Arizmendiarrieta, "J.M. " "Escuela Profesional, (E.P.)»(1997): Textos recopilados en Pensamiento de D. José María Arizmendiarrieta CD.

Arizmendiarrieta, J.M. ${ }^{a}$ "Formación Cooperativa (F.C.)» (1997): Textos recopilados en Pensamiento de D. José María Arizmendiarrieta CD.

Arizmendiarrieta, J.M. ${ }^{a}$ "Sermones, (S.S.)» (1997): Textos recopilados en Pensamiento de D. José María Arizmendiarrieta CD.

Arizmendiarrieta, J.M. " "Primeras Realizaciones, (P.R.)» (1997): Textos recopilados en Pensamiento de D. José María Arizmendiarrieta CD.

Ashord, B. y Gibbs, B. (1990): «The double-edge of organizational legitimation». Organization Science, vol. 1, n. ${ }^{\circ}$ 2, pp. 177-194.

Azpiazu, J.A. (1999): El acero de Mondragon en la época de Garibay. Ayuntamiento de Mondragón, Mondragón.

Azurmendi, J. (1987, 1992): El hombre cooperativo, pensamiento de Arizmendiarrieta. Otalora, Mondragón.

Barandiaran Contreras, M. (2000): Ampo Poyam, Elkarlanean, un recorrido en cooperativa. Litografía Danona, Oiartzun.

Barley, S.R. y Tolbert, P.S. (1997): «Institutionalization and structuration: Studying the links between action and institution", Organization Studies, vol. 18, n. ${ }^{\circ}$ 1, pp. 93-117.

Barruso Barés, P., Larrinaga Rodriguez, C., Luengo Teixidor, F., Ortiz de Orruño Legarda, J.M.a y Pérez Pérez, J.A. (2005): Historia del País Vasco. Edad Contemporánea. Hiria, Donostia.

Boons, F. y Strannegard, L. (2000): «Organizations coping with their natural environment. A laboratory for institutionalization?», International Studies of Management and Organizations, vol. 30. n. ${ }^{\circ}$ 3, pp.7-17.

Bradley, K. y Gelb, A. (1983): Cooperation at Work: the Mondragón Experience. Heinemann Educational Book, London.

Caja Laboral Popular (1967): Una Experiencia Cooperativa. Bilbao.

Cluster del Conocimiento (2003): La trayectoria de Fagor Ederlan en el exigente sector de la automoción. Cluster del Conocimiento, Zamudio.

DACIN, M.T. (1997): "Isomorphism in context. The power and prescription of institutional norms", Academy of Management Journal, vol. 40, n. ${ }^{\circ}$ 1, pp.46-81.

Del Arco, J.L. (1983): «El complejo cooperativo de Mondragón», Revesco, Estudios Cooperativos, n. 51.

DiMaggio, P.J. y Power, W.W. (1983): «The iron cage revisited: institutional isomorphism and collective rationality", American Sociological Review, vol. 48, pp. 147-160.

Elıo, E. (2004): Fagor ederlan 40 años de historia. 40 urteko historia. Idazti Servicios Editoriales, Aretxabaleta.

Fischer, R. (1975): El Complejo Cooperativo de Mondragón: estudio empirico de un sistema social. Centro de Estudios Cooperativos, Universidad Católica de Chile. Serie de estudios n. ${ }^{\circ} 10$, Santiago.

Galaskiewicz, J. (1985): "Professional networks and the institutionalization of a single mind set», American Sociological Review, vol. 50, pp. 639-658.

Garcia, Q. (1970): Les coopératives industrielles de Mondragon. Collection Économie humaine, Éditions Économie et Humanisme/Éditions Ouvrières, Paris.

Garmendia, V. (1985): La ideología carlista (186-1976). En los orígenes del nacionalismo vasco. Itxaropena, Zarautz. 
González García, J.M. (2005): La metalurgia guipuzcoana en el siglo XX: Unión Cerrajera, Patricio Echeverria, CAF, San Pedro de Elgoibar, Orbea. Industri arrastroak, Donostia.

González Portilla, M. y Garmendia, J.M. a (1988): La posquerra en el País Vasco: política, acumulación, miseria. Kriselu, Donostia.

Gorroñogoitia, A. (1984): La organización interna de una cooperativa. Otalora, Aretxabaleta.

Greenwood, D. y Gonzalez, J.L. (1990): Culturas de Fagor, estudio antropológico de las cooperativas de Mondragón. Editorial Txertoa, Donostia.

Номовоno, J.I. (1988): Caridades, cofradias y fiestas. Los santos mártires Emeterio y Celedonio de Osintxu (Bergara y Soraluce/Placencia de las Armas (Gipuzkoa). Kobie, Serie Antropología Cultural, n. ${ }^{\circ} 3$.

Номовоnо, J.I. (1991): "Ámbitos culturales, sociabilidad y grupo doméstico en el País Vasco", en Antropología de los Pueblos del Norte de España. Universidad Complutense de Madrid/Universidad de Cantabria, Madrid.

ItÇAINA, X. (2001): «Catholicisme et construction identitaire basque: retour sur le postulat d'une sécularisation achevée», Lapurdum, n. ${ }^{\circ}$ 6, pp. 353-366.

Jepperson, R.L. (1991): "Institutions, institutional effects and institutionalism», En Powell, W.W. y DiMaggio, P. (eds.), The new institutionalism in organizational analysis. The University of Chicago Press, Chicago, pp. 143-163.

Kasmir, S. (1999): El mito de Mondragón. Cooperativas, politica y clase trabajadora en una ciudad del País Vasco. Ediciones Txalaparta, Tafalla.

Lafourcade, M. (1983): «Le particularisme juridique», en J. Haritschelhar (dir.), Etre Basque. Éditions Privat, Toulouse, pp. 163-191.

Larrañaga, J. (1981): Don José M. ${ }^{a}$ Arizmendiarrieta y la experiencia Cooperativa de Mondragón. Litografía Danona S. Coop., Oiartzun.

Larrañaga, J. (1998): El cooperativismo de Mondragón, interioridades de una utopía. Otalora, Aretxabaleta.

Larrañaga, J.M. ${ }^{a}$ (1984): El socio cooperativista. Otalora, Aretxabaleta.

Leibar, J. y Azcarate, J. (1994): Historia de Eskola Politeknikoa, José M. a Arizmendiarrieta, 50 aniversario 1943-1993. Caja Laboral, Arrasate.

Leibar, J. y Ormaechea, J.M. a (1984): Don José M.a Arizmendiarrieta y sus colaboradores. Otalora, Aretxabaleta.

Maritain, J. (1966): Humanismo Integral. Carlos Lohlés ed., Buenos Aires.

Mathews, R. (1999): Jobs of Our Own: Building a Stakeholder Society. Pluto Press, Sydney.

MeYer, J. y Rowan, B. (1977): „Institutionalized organizations: formal structure as myth and ceremony", American Journal of Sociology, vol. 83, n. ${ }^{\circ}$ 2, pp. 340-363.

Molina, F. (2005): José M. a Arizmendiarrieta (1915-1976). Caja Laboral, Mondragón.

Molina, F. (2006): Fagor Electrodomésticos (1956-2006). Historia de una experiencia cooperativa. Caja Laboral, Mondragón.

Mongelos, J. (2003): «M.C.C: el desarrollo de una experiencia socio-empresarial innovadora en más de 40 años», en I. Irizar (dir.), Empresarios singulares. Cuatro testimonios, una experiencia cooperativa. Mondragon Unibertsitatea, LKS Consultores, Mondragón.

Moscovici, S. (1979): El psicoanálisis, su imagen y su público. Editorial Huemul, Buenos Aires.

North, D. (1990): Institutions, institutional change and economic performance. Political economy of institutions and decisions. Cambridge University Press, Cambridge. 
Oktubre Taldea (1987): Arrasate 1936. Una generación cortada. Arrasate.

Ormaechea, J.M. ${ }^{\text {a }}$ (1991): La Experiencia Cooperativa de Mondragón. Otalora, Aretxabaleta.

Ormaechea, J.M. ${ }^{a}$ (1997): Orígenes y claves del cooperativismo de Mondragón. Caja Laboral, Mondragón.

Ormaechea, J.M.a (2003): Medio siglo de la Experiencia Cooperativa de Mondragón. Otalora, Aretxabaleta.

Parra, L. y Aramendia, I. (2004): Arrasate, un pueblo forjado por los trabajadores. Arrasateko Udala, Mondragon.

Pérez de Calleja, A. (1989): Arizmendiarrieta, el hombre de acción. Fundación Gizabidea, Mondragón.

Perrow, Ch. (1991): Sociologia de las organizaciones. Mc Graw Hill, Madrid.

Pfeffer, J. (1992): Organizaciones y teoría de las organizaciones. Fondo de Cultura Económica, México.

Peters, B.G. (1999): Institutional theory in political science. The new institutionalism. Pinter, London and New York.

Power, W.W. y DiMaggio, P. (eds.). (1991): The new institutionalism in organization analysis. The University of Chicago, Chicago.

Riaza Ballesteros, J.M. ${ }^{a}$ (1968): Cooperativas de producción. Experiencias y futuro. Ediciones Deusto, Bilbao.

Scotт, W.R. (1994). "Institutions and organizations. Toward a theoretical synthesis», en Scott, W.R. y Meyer, J.W. (eds.): Institutional Environments and Organizations. Thousand Oaks Sage, Beverly Hills.

Scotт, W.R. (1995): Institutions and organizations. Foundations for organizational science. Sage publications, Beverly Hills.

Tolbert, P.S. y Zucker, L.G. (1983): «Institutional sources of change in the formal structure of organizations: the diffusion of civil service reform, 1889-1936", Administrative Science Quarterly, vol. 28.

T.U. LANKIDE (2003): Los fondos de intercooperacion, n. 483.

Ugalde, M. (1997): Nueva sintesis de la historia del País Vasco. Ttartalo, Donostia.

ULARCO (1975): Informe: Nuevas Formas de Organización del trabajo.

Urruela Rodriguez, V. y Urrutikoetxea Barrutia, M. (1994): «La participación en la empresa: el Grupo Mondragón», en Actas del Congreso de Sociología. Asociación Vasca de Sociología.

Whyte, W.F. y Whyte, K.K. (1989): Mondragón: más que una utopia. Editorial Txertoa, Donostia.

Zucker, L.G. (1977): «The role of institutionalization in culture persistence», American Sociological Review, vol. 42, pp. 726-743. 